\title{
Chemically Fueled Block Copolymer Self-Assembly into Transient Nanoreactors
}

\author{
Michaela A. Würbser, ${ }^{[a]}$ Patrick S. Schwarz, ${ }^{[a]}$ Jonas Heckel, ${ }^{[b]}$ Alexander M. Bergmann, ${ }^{[a]}$ Andreas \\ Walther ${ }^{*[c]}$ and Job Boekhoven ${ }^{\star[a],[d]}$
}

[a] Michaela A. Würbser, Patrick S. Schwarz, Alexander M. Bergmann, Prof. Dr. Job Boekhoven

Department of Chemistry

Technical University Munich

Lichtenbergstraße 4, 85748 Garching, Germany

[b] Jonas Heckel

Institute for Macromolecular Chemistry

University of Freiburg

Stefan-Meier-Str. 31, 79104 Freiburg, Germany

Freiburg Materials Research Center (FMF)

University of Freiburg

Stefan-Meier-Str. 21, 79104 Freiburg, Germany

Freiburg Center for Interactive Materials and Bioinspired Technologies (FIT)

University of Freiburg

Georges-Köhler-Allee 105, 79110 Freiburg, Germany

[c] Prof. Dr. Andreas Walther

A $^{3}$ BMS Lab

Department of Chemistry

University of Mainz

Duesbergweg 10-14, 55128 Mainz

Cluster of Excellence livMatS @ FIT - Freiburg Center for Interactive Materials and Bioinspired Technologies

University of Freiburg

Duesbergweg 10-14, 55128 Mainz

E-mail: andreas.walther@uni-mainz.de

[d] Prof. Dr. Job Boekhoven

Institute for Advanced Studies

Technical University Munich

Lichtenbergstraße 2a, 85748 Garching, Germany

E-mail: job.boekhoven@tum.de

Supporting information for this article is given via a link at the end of the document.

\begin{abstract}
In chemically fueled supramolecular materials, molecular self-assembly is coupled to a fuel-driven chemical reaction cycle. The fuel-dependence makes the material dynamic and endows it with exciting properties like adaptivity and autonomy. In contrast to the large work on the self-assembly of small molecules, we herein designed a diblock copolymer, which self-assembles into transient micelles when coupled to a fuel-driven chemical reaction cycle. Moreover, we used these transient block copolymer micelles to locally increase the concentration of hydrophobic reagents and thereby function as a transient nanoreactor.
\end{abstract}

\section{Introduction}

In chemically fueled self-assembly, the self-assembly of molecules is regulated by a fuel-driven chemical reaction cycle. ${ }^{[1]}$ The reaction cycle comprises at least two reactions, i.e., a fueldriven activation reaction that activates building blocks for selfassembly and a deactivation reaction that reverts the building blocks to their initial state. Thus, in response to a chemical fuel, a population of transient products emerges that can assemble into a desired morphology. Successful reaction cycles include the formation of transient methyl esters driven by the hydrolysis of methylating agents, ${ }^{[2]}$ the phosphorylation of peptides driven by the hydrolysis of ATP, ${ }^{[3]}$ ATP-assisted phosphodiester bond formation in DNA systems, ${ }^{[4]}$ and the formation of anhydrides or active esters by the hydration of carbodiimides. ${ }^{[5]}$ The emerging dynamic assemblies range from oil- or coacervated-based droplets ${ }^{[5 f, 6]}$ colloids, ${ }^{[7]}$ vesicles, ${ }^{[5 g, 8]}$ fibers, ${ }^{[2 a, ~ 2 b, ~ 5 a, ~ 5 b, ~ 5 e, ~ 9] ~ s u p r a-~}$ molecular polymers, ${ }^{[10]}$ hybridized DNA, ${ }^{[4,11]}$ clusters of nano- or microparticles ${ }^{[2 c, 5 c, 5 d]}$ and others. Due to their fuel dependence, the emerging assemblies and their material properties show a limited lifetime which can be tuned by the amount of fuel added. ${ }^{[12]}$ Exciting examples of such materials include self-erasing inks, ${ }^{[5 e, 13]}$, transient photonics, ${ }^{[14]}$ temporary hydrogels, $\left.{ }^{[5 a,} 11 \mathrm{c}, 15\right]$ transient emulsions, ${ }^{\left[{ }^{[f,}, 6 \mathrm{~d}\right]}$ and transient nanoreactors. ${ }^{[16]}$

Inspired by biological systems, nanoreactors have been developed to increase the efficiency of chemical reactions by up concentrating reactants. ${ }^{[17]}$ There are many biological examples of such nanoreactors, which can differ from simple systems, such as enzymes, to complex self-assembled structures, such as organelles or cells. ${ }^{[18]}$ Most of these structures are maintained in chemically regulated non-equilibrium states in order to control reactions spatially and temporally. ${ }^{[19]}$

Synthetic nanoreactors have been well studied under equilibrium conditions, and examples include polymer micelles, ${ }^{[20]}$ vesicles, ${ }^{[21]}$ star-shaped polymers, ${ }^{[22]}$ core-shell micelles, ${ }^{[23]}$ and dendrimers. ${ }^{[2]}$ These assemblies locally increase the concentration of substrates or catalysts. In contrast, chemically fueled examples are rather scarce. Inspired by biological systems, the in-situ production of nanoreactors in response to a 
chemical fuel offers additional control parameters over the catalyzed reaction, such as temporal and spatial control as well as control over the reaction yield. Recent work has demonstrated that the yield of a reaction can be tuned by the amount of fuel for the dissipative self-assembly of transient nanoreactors. ${ }^{[16]}$ Moreover, similar systems have been used in the context of physical autocatalysis. ${ }^{[6]}$ In this work, we thus set out to further diversify the types of self-assembly and couple a chemically fueled reaction cycle to the self-assembly of block copolymer micelles. The formed assemblies are used as transient nanoreactors to catalyze the Diels-Alder reaction of insoluble reagents in aqueous media.

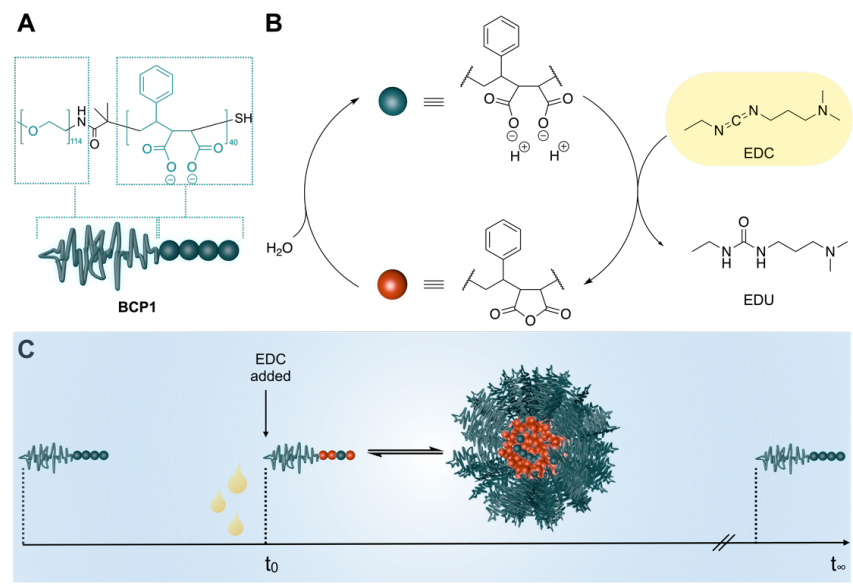

Figure 1. Chemically fueled self-assembly of BCP1 in block copolymer micelles. A) The chemical structure of diblock copolymer BCP1. B) The fuel-driven reaction cycle converts dicarboxylates-based monomers into their corresponding anhydrides. The anhydrides hydrolyze back to their precursor state. C) Schematic overview of the transient anhydride formation resulting in surfactant block copolymer products which are supposed to self-assemble into transient micelles and hydrolyze back to the precursor state after depletion of the fuel.

\section{Results and Discussion}

To create transient block copolymer-based assemblies, we designed a diblock copolymer (PEG $\left.{ }_{114}-\mathrm{b}-\mathrm{PSMA}_{40}, \mathbf{B C P 1}\right)$ that comprises a hydrophilic poly(ethylene glycol) (PEG) block and a poly(styrene-alt-maleic acid) (PSMA) block (Figure 1A, the subscripts denote the number-average degree of polymerization). The PEG block was chosen as a permanent hydrophilic block that does not change its function in response to the reaction cycle. In contrast, the PSMA block is hydrophilic by nature but can be activated by the chemical reaction cycle to become hydrophobic. We envisioned that in the fuel-driven reaction cycle, part of the maleic acid carboxylate groups of the block copolymer BCP1 react with the condensing agent 1-ethyl-3-(3-dimethylaminopropyl)carbodiimide (EDC), resulting in the formation of the corresponding cyclic anhydride (Figure 1B). We refer to this hydrophobization reaction as the activation reaction, as it activates the precursor for self-assembly. However, in the aqueous environment, these activated building blocks react back to yield the original precursor, which constitutes the deactivation reaction. Taken together, the building block is designed to transiently switch from hydrophilic to hydrophobic at the expense of a chemical fuel (Figure 1C).
We analyzed the evolution of the chemical reaction cycle with BCP1 as the precursor in response to $2.5,4.0$, and $5.0 \mathrm{mM}$ of EDC by high-pressure liquid chromatography (HPLC). In all experiments, BCP1 was used at a $5.0 \mathrm{mM}$ concentration when expressed in the concentration of maleic acid monomer units, and the water in which it was dissolved was buffered with $100 \mathrm{mM}$ MES at $\mathrm{pH}$ 6.0. We measured the rapid decay of the EDC concentration, which was fully consumed over a period of 20 minutes and could be fitted with a pseudo-second-order decay using a previously described kinetic model (see Supporting Figure S1). We measured the evolution of the anhydride concentration indirectly via a method we recently introduced. ${ }^{[25]}$ Briefly, we added benzylamine to quench the activation and deactivation reaction almost instantaneously. After the quench, we determined the converted benzylamine as a measure of the anhydride concentration (Figure 2A). The rapid decay of the EDC coincided with a rapid increase of the anhydride concentration. From there on, the anhydride decayed slowly with first-order kinetics corresponding to a half-life of roughly $82 \mathrm{~min}$. The rate constants could be combined to update a previously described kinetic model that accurately predicted the evolution of our reaction cycles for several initial concentrations of fuel (Supporting Table S2, Supporting Figure S3). ${ }^{[5]}$

Interestingly, we observed a difference in foaminess and interfacial tension of the solution, when we fueled BCP1 with EDC (Figure 2B). After shaking the solution with polymer but without EDC, we observed some foaminess which lasted around 3 hour (Figure 2B, top). In contrast, shaking the same sample with $5 \mathrm{mM}$ EDC induced more foam formation, and it remained for more than 6 hour (Figure 2B, bottom). The increased foam stability is likely a result of a slightly decreased interfacial surface tension between the air and water interface. We confirmed this by pendant drop interfacial tensiometry (Supporting Table S3), showing that the surface tension decreased from $56 \mathrm{mNm}^{-1}$ to $52 \mathrm{mNm}^{-1}$ upon fuel addition and restored after several hours.

Using cryogenic transmission electron microscopy (cryoTEM), we studied the fuel-induced changes in the solution further. Before the addition of $5 \mathrm{mM} \mathrm{EDC}$, we found no evidence of selfassembly of BCP1 (Figure 2C). Ten minutes after, the evolution of monodisperse, self-assembled micelles with a diameter of roughly $10 \mathrm{~nm}$ can be observed that disappeared after 24 hours. The emergence and decay of small self-assembling species was further confirmed by dynamic light scattering (DLS). We found a rapid increase in the scattering intensity after the addition of the fuel (Figure 2D). The hydrodynamic diameter at the maximum scattering intensity was roughly $18 \mathrm{~nm}$, further confirming the formation of micelles upon the addition of fuel (Supporting Figure S5). Over the course of the reaction cycle, we found that the scattering decreased. The decay of the scattering coincided with the decay of the anhydride concentration further corroborating the correlation between the emergence and decay of the assembly at the expense of the chemical fuel. 

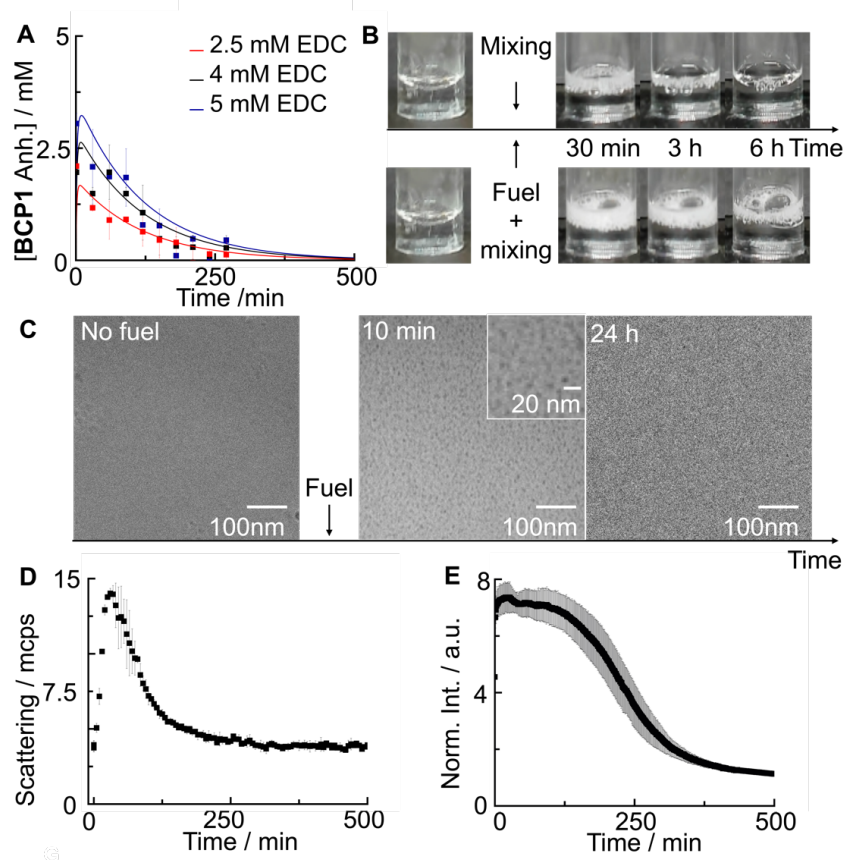

Figure 2. Transient micelle formation at the expense of a chemical fuel. A) Anhydride concentration against time when $5 \mathrm{mM} \mathrm{BCP1}$ was fueled with 2.5, 4 , or 5 mM EDC. Markers represent HPLC data; solid lines represent theoretical data calculated using the kinetic model. B) Time-lapsed photographs of the foamy solutions of $5 \mathrm{mM}$ BCP1 solution (top) and $5 \mathrm{mM}$ BCP1 solution fueled with $5 \mathrm{mM}$ EDC (bottom). The solution with EDC sustained its foam significantly longer. C) Cryo-TEM micrographs of $5 \mathrm{mM} \mathrm{BCP1}$ before, $10 \mathrm{~min}$, and $24 \mathrm{~h}$ after the addition of $5 \mathrm{mM}$ EDC. D) Scattering rate as a function of time and normalized fluorescence intensity as a function of time (E) of $5 \mathrm{mM} \mathrm{BCP1}$ fueled with $5 \mathrm{mM}$ EDC in the presence of $2.5 \mu \mathrm{M}$ Nile Red.

Furthermore, we tested the formation of hydrophobic domains by a Nile Red fluorescence assay. This assay is a convenient method to test whether hydrophobic molecules can be incorporated into assemblies (Figure 2E). ${ }^{[26]}$ Such inclusion of hydrophobic derivatives would be a requirement for the use of our transient assemblies as nanoreactors. When we fueled a solution of $5 \mathrm{mM}$ BCP1 and $2.5 \mu \mathrm{M}$ Nile Red with $5 \mathrm{mM}$ EDC, we found that the fluorescence intensity immediately increased, pointing at its uptake by the assemblies. Moreover, the fluorescence intensity decreased over the course of the reaction suggesting that Nile Red is released upon disassembly. Taken together, we successfully designed a diblock copolymer that transiently selfassembles into micelles in response to EDC as chemical fuel and is able to incorporate and release hydrophobic molecules.

Next, we tested the transient micelles' ability to partition and locally up concentrate reactants, thus serving as nanoreactors and accelerating reactions. The Diels Alder reaction is a prominent example for being catalyzed by the micelles, as this has been demonstrated before with conventional micelles and the reaction conditions are compatible with our EDC-driven cycle. ${ }^{[27]}$ A downside of using micelles as a nanoreactor can be product inhibition, i.e., the product remains in the micelles, which reduces its activity. The transient character of our micelles could prevent such product inhibition.

We combined our EDC-driven block copolymer micelles with the Diels-Alder reaction of $N$-benzyl maleimide (1) and sorbic alcohol or ethyl sorbate (2a or $\mathbf{2 b}$, respectively) which are known as suitable dienophile and dienes for the Diels-Alder reaction
(Figure 3A). ${ }^{[27 a, 27 e, 28]}$ We chose these reagents because of their difference in hydrophilicity.

We first performed the reaction with $0.2 \mathrm{mM}$ of 1 and $5 \mathrm{mM}$ of $2 \mathrm{a}$ in buffered aqueous solution and observed a fast reaction and full turnover to product 3a (see Supporting Figure S6, S7, and S10). An excess of the diene guaranteed a pseudo-first-order reaction. Upon addition of $5 \mathrm{mM} B C P 1$, the reaction remained unaffected (Figure 3B). In the presence of $30 \mathrm{mM}$ EDC, no evidence for micellar catalysis was found (Figure 3B). In fact, the reaction decelerated slightly. These findings can be explained by the relatively high solubility of $\mathbf{2 a}$ (roughly $150 \mathrm{mM}$, see Supporting Table S6). We conclude that the reaction preferably occurred in the aqueous media. In fact, partitioning of some $\mathrm{N}$-benzyl maleimide (1) in the micelles may explain the slight deceleration as it separates the reagents from one another.

Next, we performed the reaction in a buffered aqueous solution using $0.2 \mathrm{mM}$ of 1 and an excess of the more hydrophobic diene $\mathbf{2 b}(5 \mathrm{mM})$ to form the Diels Alder product $\mathbf{3 b}$. The reaction reached a poor yield of roughly $8 \%$ after 800 minutes, likely due to the low solubility of $\mathbf{2 b}$ (see Supporting Figure S8, S9 and S11). In the presence of $5 \mathrm{mM}$ of BCP1, the reaction yield remained unchanged (Figure $3 C$ ). In contrast, the yield of $\mathbf{3 b}$ increased substantially upon fuel addition. We extended our kinetic model such that it also predicted the evolution of the Diels-Alder reactions. We used a pseudo firstorder rate equation to calculate the rate every second in the reaction cycle. This reaction rate was divided into two parts, i.e., the reaction that occurred in solution $\left(k_{6}{ }^{*}[1]\right)$ and the reaction that occurred in the nanoreactor which we made dependent on the anhydride concentration of BCP1 (kcat * [1] * [Anh]) (see Supporting Notes).

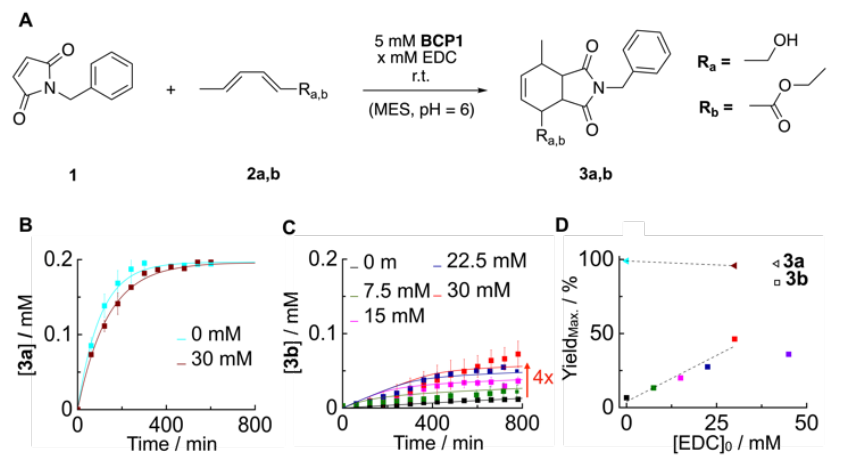

Figure 3. BCP1 micelles as dissipative nanoreactors for Diels-Alder reaction. A) Reaction scheme of the Diels-Alder reaction of the dienophile 1 with the dienes $\mathbf{2} \mathbf{a}$ and $\mathbf{2} \mathbf{b}$ to form the respective Diels-Alder products $\mathbf{3 a}$ and 3b. B) Concentration profile of 3a for $0.2 \mathrm{mM} 1$ and $5 \mathrm{mM} \mathrm{2a}$ in $5 \mathrm{mM} \mathrm{BCP1}$ solution fueled with 0 and $30 \mathrm{mM}$ EDC. The presence of BCP1 micelles has no effect on the reaction rate and yield of $\mathbf{3 a}$. C) Concentration profile of $\mathbf{3 b}$ for $0.2 \mathrm{mM} 1$ and $5 \mathrm{mM} 2 \mathrm{~b}$ in $5 \mathrm{mM} \mathrm{BCP1}$ solution fueled with different amounts of EDC. The yield increases with increasing fuel concentration. D) Maximum yield of $\mathbf{3} \mathbf{a}$ and $\mathbf{3 b}$ in dependence on the initial fuel concentration. Markers represent HPLC data; solid lines represent theoretical data calculated using the kinetic model.

We found a positive $\mathrm{k}_{\mathrm{cat}}$ when we performed the reaction of 1 and $\mathbf{2 b}$ in the presence of BCP1 and fuel (Supporting Table S9). Our kinetic model showed that the catalytic activity of BCP1 increases roughly 7.5 -fold when fully converted to the anhydride (Supporting Figure S13). Larger fuel concentrations resulted in 
higher reaction yields which we attributed to a longer sustained acceleration rate of BCP1 (Supporting Figure S13). For example, when using $30 \mathrm{mM}$ EDC, the yield of $\mathbf{3 b}$ was improved by a 4 -fold compared without EDC. When we used fuel concentrations higher than $30 \mathrm{mM}$, we observed that the yield of $\mathbf{3 b}$ did not increase further, e.g., the product evolution of $\mathbf{3 b}$ for $45 \mathrm{mM}$ EDC reached a yield of roughly $40 \%$ (see Supporting Figure S12). We explain this limitation by the hydrolysis of $\mathbf{1}, \mathbf{2 b}$ and $\mathbf{3 b}$ and the accumulation of the waste. Indeed, the behavior of the Diels-Alder reaction in the presence of sodium dodecyl sulfate (SDS), a common anionic surfactant, also increased the reaction yield to only $38 \%$ after 800 minutes (Supporting Figure S11).

We investigated how the yield is dependent on the initial fuel concentration for both reactions. For the Diels-Alder reaction of $3 \mathbf{b}$, we found that the yield increases linearly with an initial concentration of the fuel (Figure 3D). However, the increased yield leveled off at fuel concentrations higher than $30 \mathrm{mM}$. Unlike $\mathbf{2 b}$, for the Diels-Alder reaction of $\mathbf{2 a}$, the yield and reaction rate was independent of the initial fuel concentration indicating that the reaction preferably occurs in the aqueous environment.

It is worth mentioning that our kinetic model could also predict the evolution of the Diels-Alder with the well soluble $\mathbf{2 a}$ diene, provided that we used a negative $\mathrm{k}_{\mathrm{cat}}$ (Supporting Table S7) which resulted in a slower reaction rate and further corroborates that the presence of the micelles separates the reagents causing slight deceleration of the reaction. ${ }^{[29]}$ These combined results clearly demonstrate presence of the micelles can accelerate a Diels Alder reaction provided that both reactants are sufficiently hydrophobic. We can thus conclude that our micelles are not only as efficient in catalyzing Diels-Alder reactions just as regular surfactants but particularly possess the advantage of tuneability of yield or lifetime by their fuel-driven reaction cycle. Moreover, the disassembly of the micelles after depletion of the fuel prevents potential product inhibition.

\section{Conclusions}

In this work, we used a carbodiimide-fueled chemical reaction cycle to regulate the transient assembly of a diblock copolymer with fuel-switchable amphiphilicity. In response to chemical fuel, the block copolymers were transiently activated for assembly into micelles which showed a notably reduced interfacial tension between air and water. We demonstrate that the lifetime of the solution containing these micelles could be regulated by the amount of fuel added. We used the assemblies as a nanoreactor to transiently increase the concentration of reagents for a DielsAlder reaction and found that yields vastly improved with increasing amounts of fuel. In future work, we will further couple the nanoreactor catalysis to our fuel-driven reaction cycle, e.g., to alter the molecular structure of the precursor, which might result in fascinating properties, such as autocatalysis.

\section{Acknowledgments}

This research was conducted within the Max Planck School Matter to Life supported by the German Federal Ministry of Education and Research (BMBF) in collaboration with the Max Planck Society. J.B. is grateful for funding by the European
Research Council (ERC starting grant) under 852187. J.H. and A.W. acknowledge funding by the DFG under WA 3084/4-2.

Keywords: Chemically fueled self-assembly $\cdot$ Reaction cycles • block copolymer self-assembly $\bullet$ Transient nanoreactor

\section{References}

[1] a) S. De, R. Klajn, Adv. Mater. 2018, 30, 1706750; b) R. Merindol, A. Walther, Chem. Soc. Rev. 2017, 46, 5588; c) B. Rieß, R. K. Grötsch, J. Boekhoven, Chem 2020, 6, 552; d) N. Singh, G. J. M. Formon, S. De Piccoli, T. M. Hermans, Adv. Mater. 2020, 32, 1906834; e) G. Wang, S. Liu, ChemSystemsChem 2020, 2, e1900046.

[2] a) J. Boekhoven, A. M. Brizard, K. N. K. Kowlgi, G. J. M. Koper, R. Eelkema, J. H. van Esch, Angew. Chem. Int. Ed 2010, 49, 4825; b) J. Boekhoven, W. E. Hendriksen, G. J. M. Koper, R. Eelkema, J. H. van Esch, Science 2015, 349, 1075; c) B. G. P. van Ravensteijn, W. E. Hendriksen, R. Eelkema, J. H. van Esch, W. K. Kegel, J. Am. Chem. Soc. 2017, 139, 9763

[3] A. Sorrenti, J. Leira-Iglesias, A. Sato, T. M. Hermans, Nat. Commun. 2017, 8, 15899

[4] L. Heinen, A. Walther, Sci. Adv. 2019, 5, eaaw0590.

[5] a) S. P. Afrose, S. Bal, A. Chatterjee, K. Das, D. Das, Angew. Chem. Int. Ed. 2019, 58, 15783; b) K. Dai, J. R. Fores, C. Wanzke, B. Winkeljann, A. M. Bergmann, O. Lieleg, J. Boekhoven, J. Am. Chem. Soc. 2020, 142, 14142; c) R. K. Grötsch, A. Angı, Y. G. Mideksa, C. Wanzke, M. Tena-Solsona, M. J. Feige, B. Rieger, J. Boekhoven, Angew. Chem. Int. Ed. 2018, 57, 14608; d) R. K. Grötsch, C. Wanzke, M. Speckbacher, A. Angı, B. Rieger, J. Boekhoven, J. Am. Chem. Soc. 2019, 141 , 9872; e) M. Tena-Solsona, B. Rieß, R. K. Grötsch, F. C. Löhrer, C. Wanzke, B. Käsdorf, A. R. Bausch, P. MüllerBuschbaum, O. Lieleg, J. Boekhoven, Nat. Commun. 2017, 8, 15895; f) M. Tena-Solsona, C. Wanzke, B. Riess, A. R. Bausch, J. Boekhoven, Nat. Commun. 2018, 9 , 2044; g) C. Wanzke, A. Jussupow, F. Kohler, H. Dietz, V. R. Kaila, J. Boekhoven, ChemSystemsChem 2020, 2, e1900044.

[6] a) C. Donau, F. Späth, M. Sosson, B. A. Kriebisch, F. Schnitter, M. Tena-Solsona, H.-S. Kang, E. Salibi, M. Sattler, H. Mutschler, Nat. Commun. 2020, 11, 1; b) S. M. Morrow, I. Colomer, S. P. Fletcher, Nat. Commun. 2019, 10, 1; c) I. Myrgorodska, I. Colomer, S. P. Fletcher, ChemSystemsChem 2020; d) C. Wanzke, M. TenaSolsona, B. Rieß, L. Tebcharani, J. Boekhoven, Mater. Horiz. 2020, 7, 1397.

[7] B. Rieß, C. Wanzke, M. Tena-Solsona, R. K. Grötsch, C. Maity, J. Boekhoven, Soft Matter 2018, 14, 4852.

[8] a) S. Maiti, I. Fortunati, C. Ferrante, P. Scrimin, L. J. Prins, Nat. Chem. 2016, 8, 725; b) E. A. Post, S. P. Fletcher, Chem. Sci. 2020, 11, 9434.

[9] a) S. Debnath, S. Roy, R. V. Ulijn, J. Am. Chem. Soc. 2013, 135, 16789; b) B. A. Kriebisch, A. Jussupow, A. M. Bergmann, F. Kohler, H. Dietz, V. R. Kaila, J. Boekhoven, J. Am. Chem. Soc. 2020, 142, 20837; c) S. Panja, B. Dietrich, D. J. Adams, ChemSystemsChem 2020, 2, e1900038; d) E. Te Brinke, J. Groen, A. Herrmann, H. A. Heus, G. Rivas, E. Spruijt, W. T. Huck, Nat. Nanotechnol. 2018, $13,849$.

[10] a) S. Dhiman, R. Ghosh, S. J. George,

ChemSystemsChem 2020, 2, e1900042; b) J. LeiraIglesias, A. Tassoni, T. Adachi, M. Stich, T. M. Hermans, Nat. Nanotechnol. 2018, 13, 1021.

[11] a) E. Del Grosso, L. J. Prins, F. Ricci, Angew. Chem. Int. Ed. 2020, 59, 13238; b) E. Del Grosso, G. Ragazzon, L. J. Prins, F. Ricci, Angew. Chem. 2019, 131, 5638; c) L. Heinen, T. Heuser, A. Steinschulte, A. Walther, Nano Lett. 2017, 17, 4989 . 
[12] B. Rieß, J. Boekhoven, ChemNanoMat 2018, 4, 710.

[13] R. Klajn, P. J. Wesson, K. J. Bishop, B. A. Grzybowski, Angew. Chem. Int. Ed. 2009, 48, 7035.

[14] T. Heuser, R. Merindol, S. Loescher, A. Klaus, A. Walther Adv. Mater. 2017, 29, 1606842.

[15] a) S. Panja, B. Dietrich, D. J. Adams, ChemSystemsChem 2020, 2, e1900038; b) S. Ahmed, A. Chatterjee, K. Das, D. Das, Chem. Sci. 2019, 10, 7574; c) S. Panja, C.

Patterson, D. J. Adams, Macromol. Rapid Commun. 2019, 40, 1900251; d) T. Heuser, E. Weyandt, A. Walther, Angew. Chem. Int. Ed. 2015, 54, 13258; e) N. Singh, B. Lainer, G. J. M. Formon, S. De Piccoli, T. M. Hermans, J. Am. Chem. Soc. 2020, 142, 4083.

[16] a) S. Chandrabhas, S. Maiti, I. Fortunati, C. Ferrante, L. Gabrielli, L. J. Prins, Angew. Chem. 2020, 132, 22407; b) H. Che, S. Cao, J. C. M. van Hest, J. Am. Chem. Soc. 2018, 140, 5356; c) S. Maiti, I. Fortunati, C. Ferrante, P. Scrimin, L. J. Prins, Nat. Chem. 2016, 8, 725.

[17] a) D. M. Vriezema, M. Comellas Aragonès, J. A. A. W. Elemans, J. J. L. M. Cornelissen, A. E. Rowan, R. J. M. Nolte, Chem. Rev. 2005, 105, 1445; b) R. Chandrawati, M. P. van Koeverden, H. Lomas, F. Caruso, J. Phys. Chem. Lett. 2011, 2, 2639.

[18] K. Renggli, P. Baumann, K. Langowska, O. Onaca, N. Bruns, W. Meier, Adv. Funct. Mater. 2011, 21, 1241.

[19] a) R. Pascal, A. Pross, Origins Life Evol. Biospheres 2016. 46,507 ; b) C. H. Lineweaver, C. A. Egan, Physics of Life Reviews 2008, 5, 225.

[20] M. J. Monteiro, Macromolecules 2010, 43, 1159.

[21] P. Tanner, P. Baumann, R. Enea, O. Onaca, C. Palivan, W. Meier, Acc. Chem. Res. 2011, 44, 1039.

[22] H. Gao, Macromol. Rapid Commun. 2012, 33, 722.

[23] R. J. R. W. Peters, I. Louzao, J. C. M. van Hest, Chem. Sci. 2012, 3, 335.

[24] B. Rasmussen, J. B. Christensen, Org. Biomol. Chem. 2012, 10, 4821.

[25] F. Schnitter, J. Boekhoven, ChemSystemsChem 2021, 3, e2000037.

[26] J. Heckel, S. Loescher, R. T. Mathers, A. Walther, Angew. Chem. 2020.

[27] a) T. Rispens, J. B. F. N. Engberts, J. Org. Chem. 2003 68, 8520; b) T. Rispens, J. B. F. N. Engberts, J. Org. Chem. 2002, 67, 7369; c) S. Otto, J. B. Engberts, J. C. Kwak, J. Am. Chem. Soc. 1998, 120, 9517; d) P. A. Grieco, P. Garner, Z.-m. He, Tetrahedron Lett. 1983, 24, 1897; e) K. Bica, P. Gärtner, P. J. Gritsch, A. K. Ressmann, C. Schröder, R. Zirbs, Chem. Commun. 2012, 48, 5013.

[28] G. D. Khandelwal, B. L. Wedzicha, Food Chem. 1997, 60, 237.

[29] S. Otto, J. Engberts, Pure Appl. Chem. 2000, 72, 1365. 
Entry for the Table of Contents

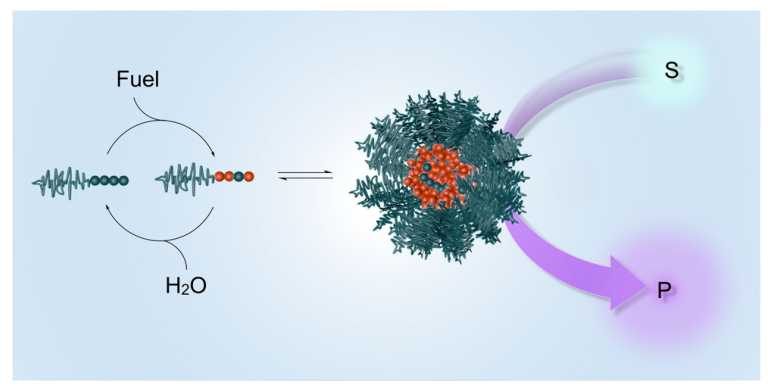

The self-assembly of block-copolymer micelles is coupled to a fuel-driven chemical reaction cycle. In their finite lifetime, the micelles are able to locally increase the concentration of reagents for a Diels-Alder reaction and drastically accelerate its rate. Thus, the kinetically controlled block copolymer micelles function as temporary nanoreactor.

Institute and/or researcher Twitter usernames: @BoekhovenLab, @WaltherLab 